\title{
Risk Management in Global Supply Chain Networks
}

\author{
N. Viswanadham and Roshan S. Gaonkar \\ The Logistics Institute - Asia Pacific, National University of Singapore, 10 Kent Ridge \\ Crescents, Singapore 119260
}

\begin{abstract}
In this paper, we develop a framework to classify the Global supply chain risk management problems and present an approach for the solution of these problems. The risk management problems need to be handled at three levels strategic, operational and tactical. In addition, risk within the supply chain might manifest itself in the form of deviations, disruptions and disasters. To handle unforeseen events in the supply chain there are two obvious approaches: (1) to design chains with built in risk-tolerance and (2) to contain the damage once the undesirable event has occurred. Both of these approaches require a clear understanding of undesirable events that may take place in the supply chain and also the associated consequences and impacts from these events. We focus our efforts on mapping out the propagation of events in the supply chain due to supplier non-performance, and employ our insight to develop a mathematical programming based model for strategic level deviation and disruption management. The first model, a simple integer quadratic optimization model, adapted from the Markowitz model, determines optimal partner selection with the objective of minimizing both the operational cost and the variability of total operational cost. This model offers a possible approach to robust supply chain design.
\end{abstract}

Key words: Supply Chain Risk Management; Risk Management; Supply Chain Planning; Supply Chain Design; Mean-Variance Optimization; Cause-Consequence Diagrams; Failure Analysis. 


\section{Introduction}

Supply chain networks are global in nature, comprising of complex interactions and flows of goods, information and funds between companies and facilities geographically distributed across countries and continents. Such chains are currently in operation in a variety of industries such as consumer electronics, automotive, pharmaceutical, aerospace, etc. Despite their complexity, most manufacturing supply chains are structurally similar. The member companies in a typical manufacturing supply chain network include the suppliers and their suppliers, assembly plants, distributors, retailers, inbound and outbound logistics providers and financing institutions. Many factors impact efficient trade in global supply chain networks: inventory visibility, late shipments, transaction costs, import and export laws compliance, customs delays, quality control problems, logistics and transport breakdowns and duplications are just a few. As China, India, Brazil, and Eastern Europe continue to grow as important economic powerhouses - with other countries becoming key second level suppliers, the cross border issues tend to dominate the supply chain management. Regulatory and compliance complexity as well as infrastructure challenges in those markets create critical supply chain and trade issues (e.g. Anti-terrorism, secure supply chain initiatives, trade facilitation etc). In fact under the intense competitive scenario prevalent today, competition is no longer between companies but between global supply chain networks with similar product offerings, serving the same global customer. The location of the supply chain constituents and the ecosystem in those countries determine the competitiveness of the supply chain. The less studied subject in the supply chain risk field is the Government risk. The antidumping duties, voluntary export restrictions are the means by which Governments would like to protect themselves against the WTO mandates and also the multilateral free trade agreements.

Because supply chain performance is inherently unpredictable and chaotic, supply chain practitioners often must seek safety mechanisms to protect against unforeseen events. Significant efforts are expended to expedite orders, to check order status at frequent intervals, to deploy inventory "justin-case" and to add safety margins to lead times. These are some of the creative ways employed to counter the occurrence of unforeseen events. These time and material inventories along with limited communications among supply chain partners hide the problems until they lead to serious consequences. Whilst risk has always been present in the process of reconciling supply with demand, there are a number of factors, which have emerged in the last decade or so, which might be considered to have increased the level of risk. These include - a focus on efficiency rather than effectiveness; the globalization of supply chains; focused factories and 
centralized distribution; the trend towards outsourcing; reduction of the supplier base; volatility of demand; lack of visibility and control procedures. As a result, it has become extremely important for channel masters to employ risk management tools in the management of their supply chains.

Supply chain risk is defined by the distribution of the loss resulting from the variation in possible supply chain outcomes, their likelihood, and their subjective values. Supply chain risks comprise risks due to variations in information, material and product flows, which originate at the original supplier and lead to the delivery of the final product to the end user. Thus supply chain risks refer to the possibility and effect of a mismatch between supply and demand. Furthermore, risk consequences can also be associated with specific supply chain outcomes like supply chain costs or quality. Within this context, we can identify the following basic constructs of supply chain risk management:

\section{Risk sources}

2. Risk consequences

3. Risk drivers

4. Risk mitigating strategies

An increased awareness of the existence of the disturbances and their sources of origin in the supply chain may enable better preparedness for handling or preventing them.

While studying risk in a supply chain network context, one also has to remember that a supply chain comprises a network of companies that belong to an industry vertical embedded in a business and social environment. Hence, supply chains are subjected to internal risks resulting from the interaction between firms within the supply chain and to external risks that are felt by all supply chain networks in the industry, and within the same environment. Consequentially supply chain risks can arise at four levels: organizational, network level, industry level and environmental level, as elaborated in Sect. 2.1. An excellent discussion on this topic may be found in Miller (1992).

In terms of existing solutions, the existing ERP, SCM, EAI and other B2B solutions are designed to improve efficiency of the supply chains and not to enhance their reliability or robustness under uncertainty. Some vendors offer partial solutions to this problem under the name of Supply Chain Event Management (SCEM). These offerings include track and trace, supply chain visibility and alert messaging solutions (Bittner, 2000), which merely notify the human operator of unexpected occurrences and leave him to resolve the issue. In such a scenario, there is a critical need for a framework and for suitable tools that would allow companies and managers 
to better understand the presence and significance of various types of risks and allow them to manage it better. In this paper we attempt to address these needs from the perspective of a channel master.

\subsection{Previous Work}

In a very general sense, research from high reliability organizations (HROs), networked organizations, and inter-organizational systems is relevant in the study of supply chain reliability, trust and risk (Grabowski et al., 2000; Grabowski and Robots; 1999). Some of the research within this area focuses on risk management in a special breed of organizations, called virtual organizations, which are also a collection of companies under independent ownership that come together for a common purpose such as fighting forest fires or mitigating the risk of oil spills.

However, in terms of directly relevant work in the area of supply chain risk management, Paulsson (2003) provides a good survey of the recent literature in the field. Some of the commonly studied supply chain risks are disruption risk, terrorism risk and the risks from natural disasters.

With reference to disruption risks, managing such risks in global supply chains includes the following procedures: identifying sources of risk, determining the means by which such risks can take place, estimating the potential consequences, and providing the approaches to mitigating and handling these consequences. Many factors can contribute to disruption risks, including natural disasters, for example, the earthquake in Taiwan in September 21, 1999 and the SARS virus outbreak in 2003, and risks arising from purposeful organizations or individuals, such as the September 11, 2001 terrorist attack and geopolitical risks. Kleindorfer and Wassenhove (2003) have also analyzed disruption risk management in global supply chains. On supply chain security, Lee and Wolfe (2003) recently discussed the strategic approaches to improving security without jeopardizing supply chain effectiveness.

In the area of terrorism risk there has been a great deal of interest especially after the September 11, 2001 terrorist attack in the U.S. Consequential to the attacks the global business environment together with the world's political and military landscape have changed greatly and companies have reassessed common strategies for sourcing transportation, demand planning and management. Sheffi (2001) studied supply chain management under the threat of international terrorism and proposed some methods such as setting certain operational redundancies. Martha and Subbakrishna (2002) also analyzed supply chains under terrorist attacks and proposed a so-called targeting a just-in-case supply chain strategy to face the inevitable next disaster. 
Another area of particular interest in supply chain risk management is that of managing risks emanating from natural disasters. Martha and Subbakrishna (2002) have investigated the impact of natural disasters on supply chains such as the earthquake in Taiwan (September 21, 1999), outbreaks of mad cow and foot and mouth diseases in Europe (Spring, 2001), and proposed the just-in-case supply chain strategy for unexpected disasters in the future. Svensson (2002) established conceptual frameworks to analyze the vulnerability in supply chains (Supply Chain Vulnerability, 2002). Svensson also provided a typology of vulnerability scenarios in supply chains based on perceived time and relationship dependencies towards both suppliers and customers (Zsidisin, 2003).

In a slightly different area one of the authors has developed a method based on process capability indices to minimize operational and performance risk through lead-time variance minimization (Garg et al., 2004). Chen and Federgruen (2000) have also, motivated by the Markowitz model studied risk management through mean-variance minimization in the context of the newsboy problem and inventory management using a base-stock policy. In addition, there are a few commercial software solutions and technology implementations to manage supply chain exceptions and events (Bittner, 2000).

Despite these publications, since the area of supply chain risk management is an emerging area of research, there are limited perspectives, theoretical models and frameworks addressing the area. We wish to provide exactly such a theoretical basis in this paper and attempt to highlight how some analytical tools can be employed to manage risk in supply chains, particularly in the context of supply risk.

\subsection{Organization of this Paper}

In this paper, we present a conceptual framework for the classification of supply chain risks and associated approaches to handling them. In particular, we focus on the design of robust supply chains at the strategic level through the selection of suppliers that minimize the variability of supply chain performance in terms of cost and output. In this manner we are able to build robustness into the supply chain at the planning stage itself. In Sect. 2, we present a conceptual framework for the classification of supply chain risks and associated approaches to building robustness in the supply chain. In Sect. 3, we develop models for supply chain risk management at the strategic level. In Sect. 4, we share some of our computational results and observations and finally we conclude in Sect. 5 with a discussion on the possibilities for future work. 


\section{Conceptual Framework to Approach Supply Chain Risk Problems}

\subsection{Nature of Risk in Supply Chains}

A number of business trends make supply networks more complex and global. Products and services are customised to better meet the demands of customers. Organisations have outsourced much of their activities to specialists allowing all to focus on their own core competencies. Internet based collaboration is blurring boundaries between manufacturing, logistics and distribution partners. All these trends make supply chains very efficient but also highly vulnerable to disruption. Network-related risk sources represent the second category of risk sources, which are the primary focus of this paper. These risks are of two broad kinds:

1. Firms are vulnerable not only to attacks on their own assets, but also to attacks on their suppliers, customers, transportation providers, communication lines, and other elements in their eco-system.

2. Firms are also vulnerable to irregular behavior of their network partners such as a supplier sharing sensitive product design with a competitor manufacturer.

In addition there are also risks for the industry as a whole. These risks could arise due to emergence of a disruptive technology or a new entrant with a sell direct kind of business model or due to input price, quality or quantity fluctuations. Environment related uncertainties affect businesses across all industries in a country or region. These include factors such as economic slow down, foreign exchange fluctuations, war, policy changes such as price controls, free trade zones, financial barriers, terrorist attacks and finally natural calamities such as earth quakes, storms, drought, etc.

\subsection{Classification of SC Risk Problems}

Based on its nature, uncertainty in the supply chain may manifest itself in three broad forms - deviation, disruption and disaster - as explained below.

\subsubsection{Deviation}

A deviation is said to have occurred when one or more parameters, such as cost, demand, lead-time, etc., within the supply chain system stray from their expected or mean value, without any changes to the underlying supply chain structure. 


\section{Examples of deviations:}

1. Variations in demand.

2. Variations in supply.

3. Variations in procurement, production and logistics costs.

4. Variations in transportation and production lead-times.

\subsubsection{Disruption}

A disruption occurs when the structure of the supply chain system is radically transformed, through the non-availability of certain production, warehousing and distribution facilities or transportation options due to unexpected events caused by human or natural factors.

\section{Examples of disruptions:}

1. Disruptions in production (Taiwan earthquake resulted in disruption of IC chip production, Component production for disrupted due to a fire in Toyota's supplier's factory in Mexico resulting in downstream factory shutdown).

2. Disruptions in supply (Meat-supply was disrupted due to spread of footand-mouth disease in England).

3. Disruptions in logistics (US port shutdown disrupted the transportation of components from Asia to the US).

\subsubsection{Disaster}

A disaster is defined as a temporary irrecoverable shut-down of the supply chain network due to unforeseen catastrophic system-wide disruptions.

\section{Examples of disasters:}

1. Terrorist Action (The entire US economy was temporarily shutdown due to the downturn in consumer spending, closure of international borders and shut-down of production facilities in the aftermath of the terrorist attacks on September 11, 2001).

2. Earth quake in a supplier country such as Taiwan.

It may be noted that the classification of an event as a disruption or a disaster is dependent on the structure of a specific supply chain and its exposure to the event. Consequently, it is very likely that a particular event might manifest itself as a disruption for one supply chain network and 
influence another in the form of a disaster. For example, the shutdown of the US trading system, subsequent to the September 11th attacks, would be a disaster for a supply chain completely based in the US. But the same event would only be a disruption for a manufacturer, located in Asia, adopting a dual-sourcing strategy for components by procuring parts both in the US and in Europe, if the manufacturer is able to keep his supply chain running by switching from US suppliers to European ones.

In general, it is possible to design a supply chain that is robust enough to profitably continue operations in the face of expected deviations and unexpected disruptions. However, it is impossible to design a supply chain network that is robust enough to react to disasters. This arises from the constraints of any system design, which is limited by its operational specification.

Furthermore, supply chains need to be robust at three levels, strategic, tactical and operational and they need to be to handle minor regular operating deviations and major disruptions at each of these three levels. For example, at the operational level, companies require decision support systems that can act on information from various partners regarding various deviations and disruptions to reschedule activities so that the business processes are synchronized and deliveries are undertaken within customer delivery windows and cost limitations. At the tactical level, plans need to have redundancies in terms of human and machine resources and also logistics and supply organizations. At the strategic level, more reliable partners with intrinsic capabilities in deviation and disruption handling, and the skills and ability to adapt to changing market conditions will be preferred and selected. A complete classification of risk management issues, with examples, at various levels and of various scopes is presented below, with examples in Table 1.

Table 1. Types of deviations

\begin{tabular}{lll}
\hline Planning level & Type of events & Example \\
\hline \multirow{2}{*}{ Strategic } & Deviation & Logistics/manufacturing capacity reduction \\
& Disruption & Supplier bankruptcy \\
\multirow{2}{*}{ Tactical } & Deviation & Order forecast \\
& Disruption & Port strike \\
\multirow{2}{*}{ Operational } & Deviation & Lead-time variation \\
& Disruption & Machine/Truck breakdown \\
\hline
\end{tabular}




\subsection{Classification of Risk Management Approaches}

Accepting the fact that uncertainty cannot be completely eliminated and given that there are several possible failure modes that can affect a supply chain network; there are two choices for building "resilient supply chains": supply chains with ability to maintain, resume and restore operations after a disruption. The first approach involves the time tested "just in case" way of maintaining inventories all along the chain, employing dual or multisourcing and manufacturing at multiple sites. This is a highly inefficient option. A better option would be to first design a sourcing strategy taking into account the disruption costs for the most relevant failure modes and then putting in place contingency plans for each disruption that include both description of the procedures to follow and a definition of roles and responsibilities. Furthermore, within this systematic approach to risk management there can be two types of responses to manage uncertainty - preventive and interceptive.

The preventive route to managing uncertainty seeks to reduce the likelihood of occurrence of an undesirable deviation or disruption through the design of a robust chain. The process starts with identifying the set of unexpected events (also commonly known as exceptions) that can occur in the chain including the interfaces. For each of these events one can conduct the root cause analysis and devise ways and means to reduce the probability of their occurrence. One can use fault trees or fish bone diagrams for doing this. This would also enable us to compute the probability of occurrence of these undesirable exceptions.

The interceptive approach on the other hands attempts to contain the loss by active intervention subsequent to the occurrence of the event (for e.g. if there is a disruption in the supply of a critical component, buy it in an exchange). This requires a very good understanding of all the available alternatives and their impact on the supply chain.

In both cases it is first necessary to identify the exceptions that can occur in the chain, estimate the probabilities of their occurrence, map out the chain of immediate and delayed consequential events that propagate through the chain and quantify their impact. In the preventive approach, the knowledge of exception probabilities and their resulting impact is employed to design chains that are inherently robust and resilient to exceptions. In the interceptive approach, once an exception occurs, based upon the map of consequential events and their impact actions that minimize the impact of the exception are initiated (Fig. 1). 


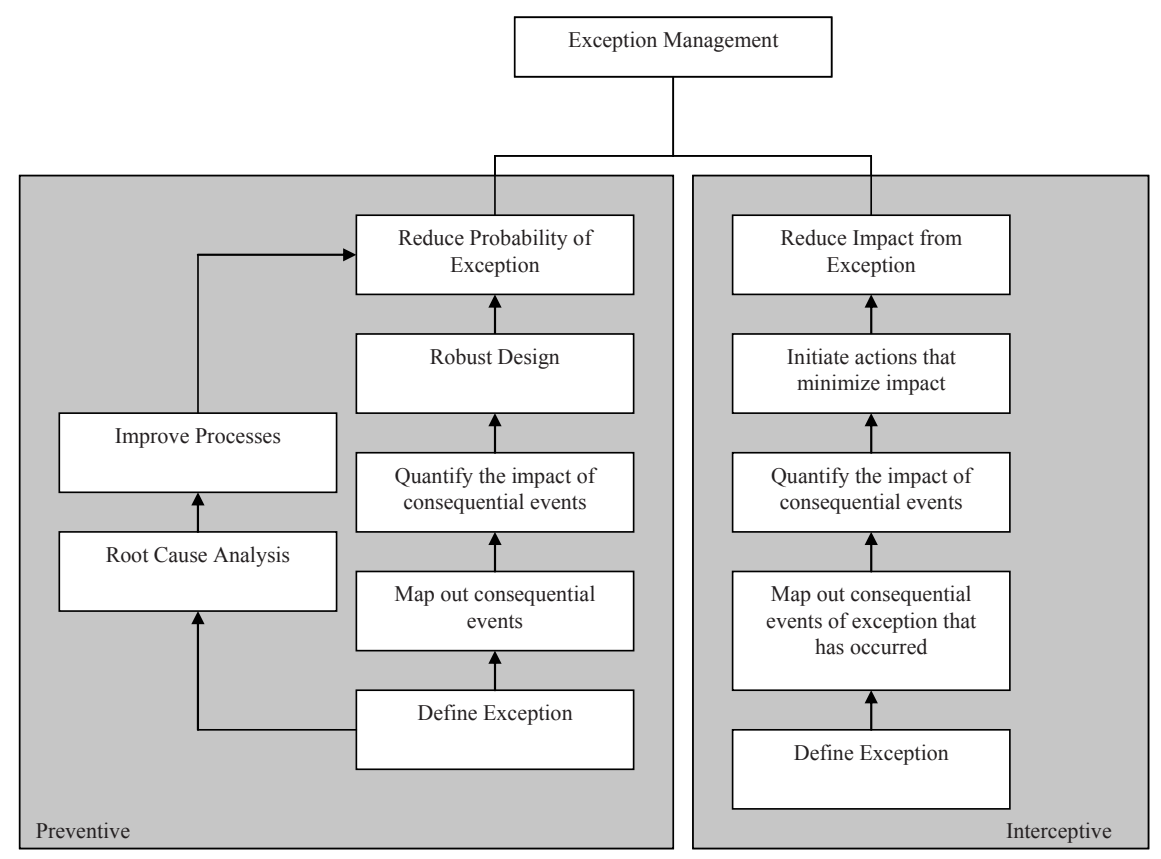

Figure 1. Exception management strategies

\subsubsection{Analytical Approaches}

Within the context of the broad classification of approaches suggested above a number of different analytical and computational methods and tools can be employed to design robust supply chains.

\subsubsection{Mathematical Planning Models}

Mathematical planning models can be employed to select and schedule processes and partners such that the overall supply chain is by design robust to internal and external stimuli. In particular, portfolio optimization models commonly applied in finance can be used to select a portfolio of suppliers such that the total supply chain cost variability and the consequences from supplier non-performance are within manageable limits, as demonstrated in the later sections of this paper. In addition, recent work in the area of robust optimization can also be used to generate supply chain solutions that maintain their optimality under minor deviations in environmental conditions. 


\subsubsection{Adaptive Control}

A multi-level adaptive control model can be built that continuously reconfigures the supply chain such that the difference between the actual and desired performance of the supply chain is minimized. The first level of an adaptive control system can be developed from a mathematical programmingbased supply chain planning model that determines optimal supply chain configurations and production and logistics schedules, which are then followed by the various participants on the supply chain. The performance of these participants is monitored and input to the second-level of the control system which then reconfigures parameters governing the first-level of the control system to provide better-designed plans that fall within the performance requirements expected from the entire supply chain. Mathematical programming models can be used to build the second-level of the control system. One such model might attempt to identify the optimal manner and location to add and deduct capacity from the supply chain such that the overall lead-times and work-in-progress inventories lie within certain specified limits. Neural networks can also be employed to build the second-level of the control system. The ensuing adaptive planning models will allow supply chains to respond in an agile manner to internal and external performance deviations.

\subsection{Basics of Uncertainty Management}

As mentioned in Sect. 2.3.1.2, for both preventive and interceptive approaches to risk management, it is necessary to identify the exceptions that can occur in the chain, estimate the probabilities of their occurrence, map out the chain of immediate and delayed consequential events that propagate through the chain and quantify their impact. In this context, it becomes important to identify the possible exceptions in a supply chain and their consequences before proceeding to the development of analytical models.

\subsubsection{Supply Chain Exception: Definition}

In attempting to analyze supply chain exceptions, our analysis here is based on a simple two-tier supply chain structure where the customer demand is directly fulfilled by a manufacturer, who in turn is supplied various components by a set of suppliers. Logistics service providers handle material movements between all the parties as shown in Fig. 2. 


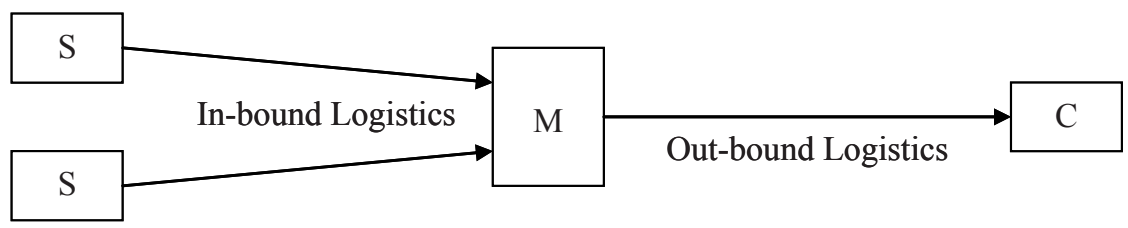

Figure 2. Simple model for analyzing exceptions

In trying to differentiate a well-executed supply chain operation from a badly managed operation we are motivated to adopt the well-accepted classical "Seven Rs" definition for the purpose of logistics, which is:

To ensure the availability of the right product, in the right quantity, in the right condition, at the right place, at the right time, at the right cost, for the right customer.

We can use this description to define a supply chain exception occurring whenever the supply chain deviates from any one of the above-required specifications either in terms of delivering the wrong product, in the wrong quantity, in the wrong condition, at the wrong place, at the wrong time, at the wrong cost and to the wrong customer. Whenever a supply chain delivery fails to stay on specification on any one of these dimensions we say that an error has been committed in that dimension.

\subsubsection{Failure or Disruption Modes}

In a supply chain exceptions can occur at various nodes - on the supply side, demand side, during transport or in storage - and due to a variety of different causes. There could be failures of power and communications or employee strikes. There is also a risk of breach of trust by partners, by outside elements.

In this paper, we specifically study supplier non-performance, in terms of the complete failure of a supplier to deliver components to the manufacturer or the inability of the supplier to deliver components at the promised price.

\subsubsection{Cause-Consequence Diagrams}

Cause-consequence diagrams or event trees are tools commonly used in reliability analysis to study the overall impact of a particular failure on the entire system. Based on the supply chain configuration, we can develop cause-consequence diagrams for each failure described above. However, given our interest in developing models for supplier selection, we employ these cause-consequence diagrams to specifically analyze the effect of 
supplier non-performance on the supply chain and to estimate the associated shortfalls in supply. For this purpose we develop the cause consequence diagram for supplier non-performance as given below in Fig. 3 .

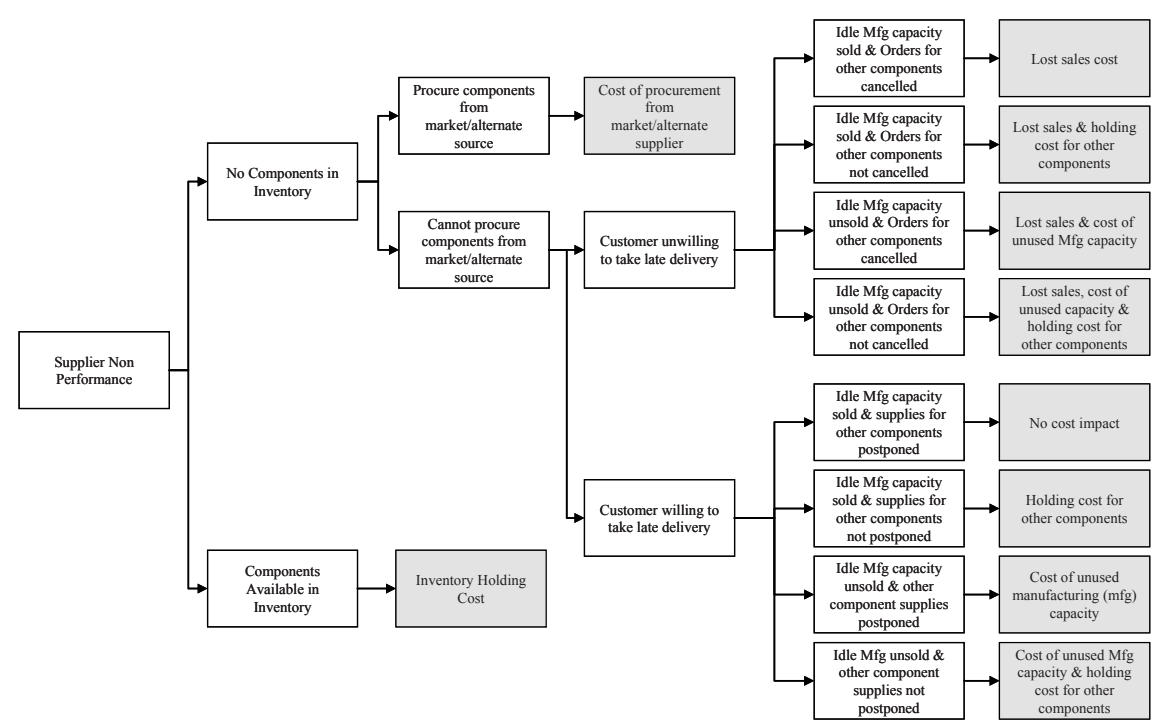

Figure 3. Cause consequence diagram for supplier non-performance and the resulting outcome

Given the probability of occurrence of the initiating event, which is supplier non-performance, and the probabilities for the various intermediary events, we can calculate the probability of occurrences for each of the end states or outcomes. Furthermore, each of these end states may result in different levels of supply shortfalls and financial cost. Hence, given the probability of each end state and the supply shortfall or financial cost for each end state, we can calculate the expected shortfall or financial risk for the non-performance of a given supplier. Such an analysis can be repeated for each supplier, and the least risky supplier can be identified as the one whose non-performance results in the least expected supply disruption or least expected financial loss.

\section{Strategic Level Supply Risk Management}

With the above foundation in the basics of supply chain risk management we now highlight the above approach by presenting two representative models for strategic level supply chain risk management, from the perspective of the 
channel master. With reference to our classification presented earlier the first model falls under the class of strategic level problems for deviation management and the second falls under the class of strategic level disruption management models. Both models employ the preventive approach to risk management based on the use of mathematical modeling techniques as described below.

1. Strategic-level Deviation Management Model: Given the expected costs and variability (deviation) of costs for all suppliers, the first problem relates to the selection of an optimal group of suppliers such that the expected cost of operating the entire supply chain and the risk of variations in total supply chain costs is minimized.

2. Strategic-level Disruption Management Model: Given the expected probabilities for various supplier disruption scenarios and the supply shortfalls under each of these scenarios the objective for the manufacturer is to choose a set of suppliers that minimize the expected shortfall during the operation of the supply chain.

It may be noted that depending on the horizon of the risk minimization and the underlying nature of the causal events the risk-parameter can be minimized using a deviation management model or a disruption management model. This choice of model will primarily depend on whether the fundamental supply chain has changed or not. If the underlying supply chain network, described by the linkages between the various supply chain participants, changes for the event studied a disruption model will be appropriate and on the other hand if the supply chain network retains its linkages a deviation model will be appropriate. This distinction between the influence of the causal events also results in certain modeling differences between the deviation management models and the disruption management models. In a deviation management model the risk-parameter to be minimized (typically a performance metric such as cost, time or demand) will invariably be modeled as a continuous variable, possibly defined by its mean and average. On the other hand, in a disruption management model the linkage impacting the supply chain network will typically be model as discrete 0 or 1 model representing the existence or absence of the linkage. It may be recognized that even while modeling disruptions the performance metrics themselves may be continuous at the system level.

In addition, for our models presented here we make the assumption that the supply chain is distributed globally and each player within the chain has its own goals, policies and cultures. The channel master who occupies a dominant position in the chain has all the information on its partners, 
including costs and schedules of the suppliers, the logistics providers, etc to be able to make a rational decision in the interest of minimizing risk.

\subsection{Strategic Level Deviation Management Model}

We propose an integer quadratic programming model for partner selection that tries to minimize the overall cost impact from the deviation in supplier costs. Such a model will be very useful to supply chain owners and channel masters. The model is an adaptation of the Markowitz model for financial portfolio management, for the purpose of managing a portfolio of suppliers. For this model, we define the impact in terms of the risk as given by the deviation of the total supply chain cost from its expected mean value. Given the expected costs and the variability of costs for all suppliers and manufacturers the objective is to choose a set of suppliers and manufacturers that minimize the expected cost of operating the entire supply chain and at the same time minimize the risk of variations in the total supply chain cost. The selection of these partners also considers the allocation of orders between these selected partners.

The mean costs and variability of the costs for each supplier can be obtained from an analysis of their historical performance or by considering the probabilities of their non-performance and the associated costs of handling the consequent impacts. Furthermore, due to the stochastic nature of events in the cause-consequence diagram we can safely assume that in general the final outcomes and associated costs of supplier non-performance will be normally distributed.

\section{Identifiers}

$\mathrm{m} \in \mathrm{M}$ : Manufacturer identifier.

$i \in I \quad:$ Component identifier.

$\mathrm{s} \in \mathrm{S}_{\mathrm{mi}}$ : Supplier identifier amongst the set of suppliers for component $\mathrm{i}$ to a specific manufacturer $\mathrm{m}$.

\section{Parameters}

$\mathrm{C}:$ Mean cost of the supply chain entity.

$\mathrm{V}$ : Cost variability for the supply chain entity.

$\mathrm{N}$ : Minimum number of entities to procure from.

$\mu$ :Risk aversion parameter $(0<\mu<\infty)$.

Large values for $\mu$ emphasize risk minimization and small values cost minimization. 


\section{Variables}

$\mathrm{X}$ : Fraction of orders and hence costs allocated between manufacturers. $(0<\mathrm{X}<1)$.

$\mathrm{Y}$ : Fraction of orders and hence costs allocated between suppliers for a specific manufacturer. $(0<\mathrm{y}<1)$.

F : 0 if supply chain entity is not selected and 1 if selected.

\section{Model}

Minimize

$$
\begin{aligned}
& \sum_{m=1}^{M} \sum_{i=1}^{I} \sum_{s=1}^{S i} y_{s} C_{s} F_{s}+\sum_{m=1}^{M} x_{m} C_{m} Y_{m} \\
& +\mu\left(\sum_{m=1}^{M} \sum_{m=1}^{I} \sum_{s=1}^{S i} y_{s}^{2} V_{s} F_{s}+\sum_{m=1}^{M} x_{m}^{2} V_{m} Y_{m}\right)
\end{aligned}
$$

Subject to

$$
\sum_{m=1}^{M} x_{m} F_{m}=1
$$

$$
\sum_{s=1}^{S_{m i}} y_{S} F_{S}=F_{m} \quad \text { forall } \quad m \in M \& i \in I
$$

$$
F_{m} \geq F_{s} \quad \text { forall } \quad m \in M \& s \in S_{m i}
$$

$$
\sum_{m=1}^{M} F_{m} \geq N_{m}
$$

$$
\sum_{S=1}^{S_{m i} F_{S} \geq N_{m i} \quad \text { forall } \quad m \in M \& i \in I}
$$

The objective of the model is to choose manufacturers and their suppliers and allocate order quantities between them in a manner such that the expected cost of operating the supply chain is minimized and also the variability of 
the overall costs is minimized as well. This is subject to the constraint that the selected set of manufacturers, between them, fulfill the order (2) and that the selected set of suppliers for these manufacturers, between them, fulfill the demand for all components (3). Suppliers are part of the supply chain only when the manufacturers they supply to are involved (4). Furthermore, there might be other policies that require a minimum number of manufacturers or suppliers to be engaged at each level of the chain for the sake of redundancy and greater reliability (5) and (6).

\subsection{Strategic Level Disruption Management Model}

With the probabilities for supplier non-performance and knowledge of supply shortfalls under various resulting end-states (as obtained from the cause-consequence diagram), we propose a mixed integer-programming model for partner selection that tries to minimize the overall impact on the supply shortfall consequential from the exception of supplier nonperformance. Such a model will be very useful to manufacturers, supply chain owners and channel masters who want to incorporate robustness into their supply chains. The model is an adaptation of the credit risk minimization model employed in financial portfolio management, for the purpose of managing a portfolio of suppliers. For this model, we define the impact in terms of the risk as given by the expected shortfall in the total supply from its expected value. Given the expected probabilities for various exception scenarios and the supply shortfalls under each of these scenarios the objective for the manufacturer is to choose a set of suppliers that minimize the expected shortfall during the operation of the supply chain.

\section{Identifiers}

$\mathrm{s} \in \mathrm{S}=$ Supplier identifier.

$\mathrm{i} \in \mathrm{I}=$ Scenario (state) identifier. $\mathrm{I}$ is the set of all supply scenarios (states), which is obtained as a mix of all combinations of supplier non-performance events for all the suppliers in the set J.

\section{Parameters}

$\mathrm{K}=$ Quantity required by the manufacturer.

$\mathrm{x}_{\mathrm{i}}=$ Quantity supplied by supplier i.

$R_{j}=$ Relation cost of including supplier $j$ into the supply chain.

$\mathrm{C}_{\mathrm{j}}=$ Capacity of supplier $\mathrm{j}$.

Variables

$F_{j}=0$ if supplier $\mathrm{j}$ is not selected and 1 if selected.

$\mathrm{y}_{\mathrm{i}}=$ Shortfall in total supply to manufacturer in scenario $\mathrm{i}$. 
Model

Minimize

$$
\sum_{i=1}^{I} p_{i} y_{i}+\sum_{S=1}^{S} R_{S} F_{S}
$$

Subject to

$$
\begin{aligned}
& K-\sum_{s=1}^{S} x_{S}=y_{i} \quad \text { forall } \quad i \in I \\
& x_{S}=F_{S} * C_{S} \quad \text { forall } \quad s \in S
\end{aligned}
$$

The objective of the model is to choose suppliers such that the expected shortfall in supply, in the face of supplier disruptions is minimized. This is subject to the constraint (2) which calculates the shortfall for each possible supply scenario. Also, the quantity supplied by any supplier is dependent on its capacity and also on the decision whether or not the supplier is included into the supply chain network (3). When the supplier is included into the supply chain network his supplies are equivalent to his capacity. This may be visualized as representing the capacity that is contracted or is expected to be contracted with the supplier.

\section{Computational Results}

For representative purposes, both the models described above were formulated in Microsoft Excel and solved using the Solver add-in.

\subsection{Strategic-Level Deviation Management Model}

This model was solved for a problem with five manufacturers, dealing with five suppliers each, for each of the two components required in their manufacturing. The risk aversion factor was taken as 25 and it was required that at least two manufacturers be selected for fulfilling the orders (Table 2).

Due to the non-linear nature of the problem, the final solution obtained depends very much on the initial values of the variables. Moreover, the choice of manufacturers is the most critical decision since it also decides to a large extent the choice of suppliers. Hence, the model was solved for various initial solutions corresponding to all the possible combinations of supplier selection. The optimal solution obtained as a result is given below (Table 3). 
Table 2. Cost and variance of cost for each partner

\begin{tabular}{|c|c|c|c|c|c|c|c|c|}
\hline \multicolumn{3}{|c|}{ Manufacturer } & \multicolumn{3}{|c|}{ Component 1} & \multicolumn{3}{|c|}{ Component 2} \\
\hline Sup & $\mathrm{C}$ & $\mathrm{V}$ & Sup & $\mathrm{C}$ & $\mathrm{V}$ & Sup & $\mathrm{C}$ & $\mathrm{V}$ \\
\hline \multirow{5}{*}{ Mfg 1} & \multirow{5}{*}{90} & \multirow{5}{*}{8} & S1 & 10 & 4 & S1 & 44 & 7 \\
\hline & & & S2 & 15 & 3 & $\mathrm{~S} 2$ & 45 & 6 \\
\hline & & & S3 & 25 & 1 & S3 & 47 & 5 \\
\hline & & & S4 & 20 & 2 & S4 & 43 & 6 \\
\hline & & & S5 & 12 & 2 & S5 & 45 & 6 \\
\hline \multirow{5}{*}{ Mfg 2} & \multirow{5}{*}{81} & \multirow{5}{*}{7} & S1 & 13 & 3 & $\mathrm{~S} 1$ & 50 & 4 \\
\hline & & & S2 & 17 & 2 & $\mathrm{~S} 2$ & 45 & 6 \\
\hline & & & S3 & 19 & 1 & S3 & 44 & 6 \\
\hline & & & S4 & 15 & 3 & S4 & 47 & 5 \\
\hline & & & S5 & 10 & 3 & S5 & 43 & 7 \\
\hline \multirow{5}{*}{ Mfg 3} & \multirow{5}{*}{84} & \multirow{5}{*}{8} & S1 & 14 & 2 & S1 & 42 & 7 \\
\hline & & & $\mathrm{S} 2$ & 16 & 3 & $\mathrm{~S} 2$ & 46 & 5 \\
\hline & & & S3 & 15 & 2 & S3 & 49 & 4 \\
\hline & & & S4 & 11 & 4 & S4 & 48 & 4 \\
\hline & & & S5 & 15 & 2 & S5 & 44 & 6 \\
\hline \multirow{5}{*}{ Mfg 4} & \multirow{5}{*}{93} & \multirow{5}{*}{6} & S1 & 12 & 3 & S1 & 45 & 5 \\
\hline & & & $\mathrm{S} 2$ & 10 & 3 & $\mathrm{~S} 2$ & 45 & 6 \\
\hline & & & S3 & 20 & 3 & S3 & 48 & 4 \\
\hline & & & S4 & 19 & 2 & S4 & 46 & 6 \\
\hline & & & S5 & 18 & 2 & S5 & 50 & 3 \\
\hline \multirow{5}{*}{ Mfg 5} & \multirow{5}{*}{99} & \multirow{5}{*}{5} & S1 & 16 & 2 & S1 & 48 & 5 \\
\hline & & & S2 & 18 & 2 & S2 & 47 & 6 \\
\hline & & & S3 & 21 & 1 & S3 & 51 & 4 \\
\hline & & & S4 & 14 & 2 & S4 & 51 & 5 \\
\hline & & & S5 & 12 & 3 & S5 & 48 & 5 \\
\hline
\end{tabular}

$C$ Mean cost; $V$ Variance of cost; $M f g$ Manufacturer; Sup Supplier

Table 3. Cost and variance of cost for each partner

\begin{tabular}{llllll}
\hline Manufacturers & & Component 1 & & Component 2 \\
\hline Mfg selected & Share & Sup & Share & Sup & Share \\
\hline \multirow{4}{*}{ Mfg 4 } & \multirow{3}{*}{0.46} & S1 & 0.167 & S1 & 0.179 \\
\cline { 3 - 6 } & & S2 & 0.167 & S2 & 0.149 \\
& S3 & 0.167 & S3 & 0.224 \\
& S4 & 0.25 & S4 & 0.149 \\
& S5 & 0.25 & S5 & 0.299 \\
\hline \multirow{3}{*}{ Mfg 5 } & S1 & 0.176 & S1 & 0.197 \\
& \multirow{2}{*}{0.54} & S2 & 0.176 & S2 & 0.164 \\
& & S3 & 0.353 & S3 & 0.246 \\
& S4 & 0.176 & S4 & 0.197 \\
\hline
\end{tabular}

Sup Supplier selected,

Share $=$ Fractional allocation of demand. 


\subsection{Strategic-Level Disruption Management Model}

This model was solved for a problem with a single manufacturer (located in the US), dealing with five suppliers. The probabilities of supplier disruption for all the suppliers (individually and in various combination) were considered as given. The relation cost was taken as $\$ 5,000$ and the quantity required by the manufacturer was 520 units. The location, capacities and risks faced for each of the suppliers is listed below in Table 4.

Table 4. Supplier pool

\begin{tabular}{llll}
\hline Supplier & Location & Capacities & Risks exposed to \\
\hline Supplier 1 & Ireland & 250 & $\begin{array}{l}\text { Terrorist Attacks } \\
\text { Union Strikes } \\
\text { Earthquakes }\end{array}$ \\
Supplier 2 & Taiwan & 250 & $\begin{array}{l}\text { US East Coast Port Closure } \\
\text { Lower Quality (Non-reliable) } \\
\text { US East Coast Port Closure }\end{array}$ \\
Supplier 3 & Malaysia & 280 & US East Coast Port Closure \\
Supplier 4 & Singapore & 340 & \\
Supplier 5 & USA & 250 & \\
\hline
\end{tabular}

As may be seen from Table 5, the third supplier is a non-reliable supplier based in Malaysia and the fourth a reliable supplier in Singapore, both of whom are susceptible to the risk resulting from closure of US ports. The fifth supplier is assumed to be a local supplier and is exposed to relatively insignificant risks as compared to the other four overseas-based suppliers. Based on the above characteristics of the various suppliers, the probabilities for various disruption scenarios were calculated in Table 5. Due to the lack of real-world data, our calculations are based on simulated data. However, it should be possible to perform the same analysis with detailed practical data such a country risk index and supplier rating data.

Table 5. Probabilities of various supply situations

\begin{tabular}{cll}
\hline Scenarios & Explanation & Probability \\
\hline 1 & Supplier 1 disrupted & 0.05 \\
2 & Supplier 2 disrupted & 0.04 \\
3 & Supplier 3 disrupted & 0.08 \\
4 & Supplier 4 disrupted & 0.01 \\
5 & Supplier 5 disrupted & 0.02 \\
6 & Suppliers 1 and 2 disrupted & 0.0015 \\
7 & Suppliers 1 and 3 disrupted & 0.0015 \\
8 & Suppliers 1 and 4 disrupted & 0.0005 \\
9 & Suppliers 1 and 5 disrupted & 0.0015 \\
10 & Suppliers 2 and 3 disrupted & 0.0016 \\
11 & Suppliers 2 and 4 disrupted & 0.0004 \\
\hline
\end{tabular}




\begin{tabular}{lll}
\hline Scenarios & Explanation & Probability \\
\hline 12 & Suppliers 2 and 5 disrupted & 0.0008 \\
13 & Suppliers 3 and 4 disrupted & 0.0008 \\
14 & Suppliers 3 and 5 disrupted & 0.0048 \\
15 & Suppliers 4 and 5 disrupted & 0.0001 \\
16 & Suppliers 1, 2 and 3 disrupted & 0.0045 \\
17 & Suppliers 1, 2 and 4 disrupted & 0.0015 \\
18 & Suppliers 1, 2 and 5 disrupted & 0.0045 \\
19 & Suppliers 1, 3 and 4 disrupted & 0.0015 \\
20 & Suppliers 1, 3 and 5 disrupted & 0.0045 \\
21 & Suppliers 1, 4 and 5 disrupted & 0.0015 \\
22 & Suppliers 2, 3 and 4 disrupted & 0.0016 \\
23 & Suppliers 2, 3 and 5 disrupted & 0.0032 \\
24 & Suppliers 2, 4 and 5 disrupted & 0.0008 \\
25 & Suppliers 3, 4 and 5 disrupted & 0.0048 \\
26 & Suppliers 1, 2, 3 and 4 disrupted & 0.000045 \\
27 & Suppliers 1, 2, 3 and 5 disrupted & 0.000135 \\
28 & Suppliers 1, 2, 4 and 5 disrupted & 0.000045 \\
29 & Suppliers 1, 3, 4 and 5 disrupted & 0.000045 \\
30 & Suppliers 2, 3, 4 and 5 disrupted & 0.000032 \\
31 & All suppliers disrupted & 0.00000135 \\
32 & None disrupted & 0.75779665 \\
\hline
\end{tabular}

The model was solved with the above data. The optimal selection of suppliers included Suppliers 4 and 5, with an objective value of 10,017. It might be noticed that these two suppliers are the most reliable suppliers.

\section{Conclusion}

We have developed a conceptual framework for the classification of risks in global supply chain networks and approaches for mitigating them. In the examples, we focus on the design of robust inbound supply chains, at the strategic level, that are resilient to deviations and disruptions that may occur at the supplier end. Our analysis is based on the identification of unforeseen events that may occur at the supplier end propagate down the supply chain leading to cost variability and supply shortfalls. Robustness is build into our supply chain design by selecting a portfolio of suppliers that minimize the variability of supply chain performance in terms of cost and output. This analysis can be extended to include other exceptions such as import and export compliance. Finally we may mention that our approach of mapping of exceptions and consequences using fault trees and event trees can form the foundation for building decision support systems for exception management in global supply chain networks. 


\section{References}

M. Bittner, E-Business Requires Supply Chain Event Management, AMR Research, November 2000.

M. Grabowski, J.R.W. Merrick, J.R. Harrald, T.A. Mazzuchi and J.R. van Dorp, Risk Modelling in Distributed, Large-Scale Systems, IEEE Transactions on Systems, Man and Cybernetics, 30 (6), November 2000.

M. Grabowski and K.H. Robots, Risk Mitigation in Virtual Organisations, Organisation Science, 10 (6), Nov-Dec 1999.

U. Paulsson, Managing Risks in Supply Chains - An Article Review, Presented at NOFOMA 2003, Oulu, Finland, 12-13 June 2003.

Y. Sheffi, Supply Chain Management under the Threat of International Terrorism, The International Journal of Logistics Management, 12 (2), 1-11, 2001.

D. Garg, Y. Narahari, and N. Viswanadham, Design of Six Sigma Supply Chains, to appear in IEEE Transactions on Automation Science and Engineering (first issue), April 2004.

M.E. Johnson, Learning From Toys: Lessons in Managing Supply Chain Risk from the Toy Industry, California Management Review, 43, 106-124, 2001.

P.R. Kleindorfer and L.V. Wassenhove, Managing Risk in Global Supply Chains, Wharton Insurance and Risk Management Department Seminar, University of Pennsylvania, February, 2003.

H.L. Lee and M. Wolfe, Supply Chain Security without Tears, Supply Chain Management Review, Jan/Feb, 12-20, 2003.

Kent D. Miller, A framework for Integrated Risk Management in International Business, Journal of International Business Studies, second quarter, 311-331, 1992.

J. Martha and S. Subbakrishna, Targeting a Just-in-case Supply Chain for the Inevitable Next Disaster, Supply Chain Management Review, Sep/Oct, 18-23, 2002.

Supply Chain Vulnerability (2002). Cranfield University, UK. January, 2002.

G. Svensson, A Typology of Vulnerability Scenarios towards Suppliers and Customer in Supply Chains Based upon Perceived Time and Relationship Dependencies, International Journal of Physical Distribution \& Logistics Management, 32, 168-187, 2002.

G.A. Zsidisin, Managerial Perceptions of Supply Risk, Journal of Supply Chain Management, 39, 14-25, 2003.

F. Chen and A. Federgruen, Mean-Variance Analysis of Basic Inventory Models, Working paper, Graduate School of Business, Columbia University, July 6, 2000.

H. Markowitz, Portfolio Selection: Efficient Diversification of Investment, Cowles Foundation Monograph 16, Yale University Press, New York, 1959. 